\title{
Measurement of the magnetic field errors on TCV
}

\author{
F. Piras*, J-M. Moret, J. X. Rossel and the TCV team \\ Ecole Polytechnique Fédérale de Lausanne (EPFL) \\ Centre de Recherches en Physique des Plasmas (CRPP) \\ Association Euratom-Confédération Suisse \\ Station 13, CH-1015 Lausanne, Switzerland
}

\begin{abstract}
A set of 24 saddle loops is used on the Tokamak à Configuration Variable (TCV) to measure the radial magnetic flux at different toroidal and vertical positions. The new system is calibrated together with the standard magnetic diagnostics on TCV. Based on the results of this calibration, the effective current in the poloidal field coils and their position is computed. These corrections are then used to compute the distribution of the error field inside the vacuum vessel for a typical TCV discharge.

Since the saddle loops measure the magnetic flux at different toroidal positions, the non-axisymmetric error field is also estimated and correlated to a shift or a tilt of the poloidal field coils.
\end{abstract}

Key words: Tokamak, Error fields, Locked modes, TCV

\section{Introduction}

In Tokamak devices plasma is confined with a magnetic field. The spacial distribution of the field defines the plasma position, the plasma shape and, in diverted plasmas, the topology of the $\mathrm{X}$-point and the position of the strike points $[1,2,3]$. During the plasma creation, the magnetic structure is a key feature to obtain a stable and repeatable breakdown [4].

To control the magnetic field distribution, it is important to know precisely the position and the electric current in each coil. Moreover, the real-time control system of the plasma parameters and the magnetic equilibrium reconstruction are mainly based on the magnetic measurements. The quality of the observers used as input for the control system and of the offline magnetic analysis is therefore imposing important constraints on the required precision of the magnetic system in a tokamak.

The axisymmetric magnetic field errors, i.e. having a toroidal mode number $n=0$, lead to a deformation of the plasma shape, wrong plasma position and degradation of the null point at the breakdown. They are due to errors in the radius and vertical position of the Poloidal Field (PF) coils and their measured currents.

\footnotetext{
*Corresponding author. tel.: +41-2169-36548; fax:+41-2169-35176

Email address: francesco.piras@epfl.ch (F. Piras)

Preprint submitted to Fusion Engineering and Design
} 
Misalignments and deformations of the PF coils are causing non-axisymmetric error fields $(n>0)[5,6,7]$. These toroidal asymmetries are often responsible for the creation of magnetic islands inside the plasma and locked modes $[8,9,10,11,12,13]$ and therefore reduction of the plasma confinement. During the breakdown, breaking of the toroidal symmetry leads to a reduction of the connection length of the magnetic field lines and the probability to obtain a stable and reproducible breakdown is therefore decreased.

The axisymmetric error field on TCV [1] has been described in detail in [14]. The whole magnetic system has been calibrated and the errors on the radial and vertical position of the PF coils and of the measured currents were computed.

A set of 24 saddle flux loops (SLs) is now used on TCV to measure the radial magnetic flux at different toroidal and vertical positions. This new magnetic diagnostic is implemented in the calibration process already described in [14] and used to obtain the $n=1$ error field on TCV. The misalignments or tilts of the PF coils are then estimated from the computed error field.

This paper is organized as follows. The TCV magnetic system, including the SLs, is described in section 2. The calibration method is summarized in section 3 and the results are discussed in section 4 . In the last section, the magnetic field inside the vacuum vessel is computed for a typical TCV discharge.

\section{The TCV magnetic system}

In Fig. 1 the cross section of TCV is shown together with the magnetic sensors. Two independent ohmic circuits are used to induce the plasma current. The O1 circuit comprises the A coil and the $\mathrm{O} 2$ circuit the coils B, C and D connected in series. Plasma shape and position are controlled with the $16 \mathrm{PF}$ coils labeled $\mathrm{E}$ and $\mathrm{F}$, powered by independent power supplies. The toroidal field is produced by 16 coils surrounding the vacuum vessel and connected together in series by the connections $\mathrm{T}$ in Fig. 1 . Since the current distribution in the connections $\mathrm{T}$ creates a poloidal magnetic field inside the vacuum vessel, the $\mathrm{T}$ coils are considered as part of the $\mathrm{PF}$ coil system.

The standard magnetic system on TCV is based on 4 arrays of 38 magnetic field probes measuring the poloidal magnetic field tangential to the vacuum vessel. These arrays are toroidally separated by $90^{\circ}$. The signals from two opposite arrays are averaged to cancel the toroidal asymmetries. The poloidal flux is measured on TCV with 61 flux loops placed around the vacuum vessel and close to the PF coils. Because of the presence of the ports, not all the vessel flux loops have a constant radius. Only a selection of 48 flux loops that do not bypass any port are considered.

The SL system (also shown in Fig. 1) is comprised of 24 SLs located at three different vertical levels denoted TOP, MIDDLE and BOTTOM, as shown in Fig. 2. Their vertical extension is given by 6 virtual poloidal flux loops $\left(\mathrm{ST}_{1,2}, \mathrm{SM}_{1,2}\right.$ and $\mathrm{SB}_{1,2}$ in Fig. 2). Each of them cover a toroidal angle of $45^{\circ}$, corresponding to an extension of two TCV sectors. They are made of one turn of $1 \mathrm{~mm}$ diameter mineral insulated coaxial wire (THERMOCOAX ${ }^{\circledR}$ ) and they are fixed to the outer surface of the vacuum vessel. On each SL, the induced voltage is proportional to the first time-derivative of the radial magnetic flux in the SL surface:

$$
v_{S L}=-\frac{\mathrm{d} \psi_{S L}}{\mathrm{~d} t}=-\int_{2} \dot{\mathbf{b}} \mathrm{d} \boldsymbol{\Omega}
$$


where $\psi_{S L}$ is the flux in the SL, $\mathbf{b}$ is the first time-derivative of the magnetic field and $\Omega_{S L}$ is the SL surface.

Each SL signal is pre-amplified near the machine to limit the impact of the long transmission line. They are then either amplified to be used as time derivatives of the magnetic flux or integrated to obtain the flux itself. An over-voltage protection is inserted between the pre-amplifiers and the amplifiers to protect the circuits against spikes induced by the plasma dynamics. The resulting signals are then filtered and acquired or used by the real time control system. A different filter is used by the real time shape and position control with a higher cut-off frequency to avoid a too large phase lag that would impair fast instability feedback.

The block diagram of the signal processing is shown in Fig. 3. All the electronic devices have been calibrated offline using the method previously described in [14].

\section{The calibration method}

The magnetic field error in TCV is evaluated using an extension of the procedure explained in [14]. In this paragraph, the method is summarized emphasizing the new features introduced by the SL signals.

Each poloidal field coil is separately powered with a given current. The typical wave form used has a flat-top of $20 \mathrm{kA}$ for the ohmic coils (O1 and $\mathrm{O} 2)$ and the toroidal field coils and $4 \mathrm{kA}$ for the other coils (E and F). During the flat-top phase, the current is controlled within $10 \mathrm{~A}$ for $1 \mathrm{~s}$. The stationary phase is longer than the vessel time constant $(\sim 13.4 \mathrm{~ms})$ to ensure that the eddy currents in the vessel and in the mechanical structures vanish. For each powered coil, all the magnetic signals are acquired and time averaged on the flat-top phase to reduce the noise and the statistical error. The measured quantities are then compared with the expected values, yielding the vector of the differences between the experimental values and the theoretical predictions:

$$
\begin{aligned}
\Delta \boldsymbol{\Psi}_{f} & =\boldsymbol{\Psi}_{f}-\underline{\underline{\mathbf{M}}}_{f c} \mathbf{I}_{\mathbf{c}} \\
\boldsymbol{\Delta} \mathbf{b}_{m} & =\mathbf{b}_{m}-\underline{\underline{\mathbf{B}}}_{m c} \mathbf{I}_{\mathbf{c}} \\
\boldsymbol{\Delta} \boldsymbol{\Psi}_{s} & =\boldsymbol{\Psi}_{s}-\underline{\underline{\mathbf{M}}}_{s c} \mathbf{I}_{\mathbf{c}}
\end{aligned}
$$

$\boldsymbol{\Psi}_{f}, \mathbf{b}_{m}$ and $\boldsymbol{\Psi}_{s}$ are the vectors of the measured quantities in the flux loops, magnetic probes and SLs respectively; $\underline{\underline{\mathbf{M}}}_{f c}, \underline{\underline{\mathbf{B}}}_{m c}$ and $\underline{\underline{\mathbf{M}}}_{s c}$ are the matrices of the Green's functions between the PF coils and the considered sensors and $\mathbf{I}_{\mathbf{c}}$ is the vector of the coil currents. Notice that $\mathbf{I}_{c}=\underline{\underline{\mathbf{T}}}_{c a} \mathbf{I}_{a}$, where $\mathbf{I}_{a}$ is the current in the combined PF circuits and $\underline{\underline{\mathbf{T}}}_{c a}$ is the transfer matrix used to obtain the current in each coil from the current in each circuit. In the same manner $\underline{\underline{\mathbf{M}}}_{f a}=\underline{\underline{\mathbf{M}}}_{f c} \underline{\underline{\mathbf{T}}}_{c a}$, where $\underline{\underline{\mathbf{M}}}_{f a}$ is the matrix of the Green's functions between the PF coil systems and the flux loops. Similarly $\underline{\underline{\mathbf{B}}}_{m a}=\underline{\underline{\mathbf{B}}}_{m c} \underline{\underline{\mathbf{T}}}_{c a}$ and $\underline{\underline{\mathbf{M}}}_{s a}=\underline{\underline{\mathbf{M}}} s \underline{\underline{\mathbf{T}}} \underline{\underline{T}}$.

The difference between the measured quantities and the expected values in Eqs. 2-4 is due to the errors in the geometrical characteristics of the PF coils, in the magnetic sensors and in the electronic devices used to acquire the signals.

Mathematically, this discrepancy can be expressed with a first order Taylor expansion as follow: 


$$
\begin{aligned}
& \boldsymbol{\Delta} \boldsymbol{\Psi}_{f}=\left(\underline{\underline{\mathbf{M}}}_{f a} \boldsymbol{\Delta} \mathbf{G}_{\mathbf{a}}+\frac{\partial \underline{\underline{\mathbf{M}}}_{f c}}{\partial R_{c}} \boldsymbol{\Delta} \mathbf{R}_{\mathbf{c}} \underline{\underline{\mathbf{T}}}_{c a}+\frac{\partial \underline{\underline{\mathbf{M}}}_{f c}}{\partial Z_{c}} \boldsymbol{\Delta} \mathbf{Z}_{\mathbf{c}} \underline{\underline{\mathbf{T}}}_{c a}\right. \\
& +\underline{\underline{\mathbf{M}}}_{f c} \frac{\partial \underline{\underline{\mathbf{T}}}_{c a}}{\partial \beta_{T}} \boldsymbol{\Delta} \beta_{\mathbf{T}}+\Delta \mathbf{G}_{\mathbf{f}} \underline{\underline{\mathbf{M}}}_{f a} \\
& \left.+\Delta \mathbf{R}_{\mathbf{f}} \frac{\partial \underline{\underline{\mathbf{M}}}_{f a}}{\partial R_{f}}+\Delta \mathbf{Z}_{\mathbf{f}} \frac{\partial \underline{\underline{\mathbf{M}}}_{f a}}{\partial Z_{f}}\right) \mathbf{I}_{a} \\
& \boldsymbol{\Delta} \mathbf{b}_{m}=\left(\underline{\underline{\mathbf{B}}}_{m a} \boldsymbol{\Delta} \mathbf{G}_{\mathbf{a}}+\frac{\partial \underline{\underline{\mathbf{B}}}_{m c}}{\partial R_{c}} \boldsymbol{\Delta} \mathbf{R}_{\mathbf{c}} \underline{\underline{\mathbf{T}}}_{c a}+\frac{\partial \underline{\underline{\mathbf{B}}}_{m c}}{\partial Z_{c}} \boldsymbol{\Delta} \mathbf{Z}_{\mathbf{c}} \underline{\underline{\mathbf{T}}}_{c a}\right. \\
& +\underline{\underline{\mathbf{B}}}_{m c} \frac{\partial \underline{\underline{\mathbf{T}}}_{c a}}{\partial \beta_{T}} \boldsymbol{\Delta} \beta_{\mathbf{T}}+\Delta \mathbf{G}_{\mathbf{m}} \underline{\underline{\mathbf{B}}}_{m a}+\Delta \mathbf{R}_{\mathbf{m}} \frac{\partial \underline{\underline{\mathbf{B}}}_{m a}}{\partial R_{m}} \\
& \left.+\Delta \mathbf{Z}_{\mathbf{m}} \frac{\partial \underline{\underline{\mathbf{B}}}_{m a}}{\partial Z_{m}}+\Delta \alpha_{\mathbf{m}} \frac{\partial \underline{\underline{\underline{B}}}_{m a}}{\partial \alpha_{m}}\right) \mathbf{I}_{a} \\
& \boldsymbol{\Delta} \boldsymbol{\Psi}_{s}=\left(\underline{\underline{\mathbf{M}}}_{s a} \boldsymbol{\Delta} \mathbf{G}_{\mathbf{a}}+\frac{\partial \underline{\underline{\mathbf{M}}}_{s c}}{\partial R_{c}} \boldsymbol{\Delta} \mathbf{R}_{\mathbf{c}} \underline{\underline{\mathbf{T}}}_{c a}+\frac{\partial \underline{\underline{\underline{\mathbf{M}}}} s c}{\partial Z_{c}} \Delta \mathbf{Z}_{\mathbf{c}} \underline{\underline{\mathbf{T}}}_{c a}\right. \\
& +\frac{\partial \underline{\underline{\mathbf{M}}}_{s c}}{\partial X_{c}} \boldsymbol{\Delta} \mathbf{X}_{\mathbf{c}} \underline{\underline{\mathbf{T}}}_{c a}+\frac{\partial \underline{\underline{\mathbf{M}}}_{s c}}{\partial \mathbf{M}} \boldsymbol{\Delta} \mathbf{Y}_{\mathbf{c}} \underline{\underline{\mathbf{T}}}_{c a} \\
& +\frac{\partial \underline{\underline{\mathbf{M}}}_{s c}^{c}}{\partial \alpha_{c x}} \boldsymbol{\Delta} \alpha_{\mathbf{c x}} \underline{\underline{\mathbf{T}}}_{c a}+\frac{\partial \underline{\underline{\underline{\mathbf{M}}}}_{s c}}{\partial \alpha_{c y}} \boldsymbol{\Delta} \alpha_{\mathbf{c y}} \underline{\underline{\mathbf{T}}}_{c a} \\
& +\underline{\underline{\mathbf{M}}}_{s c} \frac{\partial \underline{\underline{\mathbf{T}}}_{c a}}{\partial \beta_{T}} \boldsymbol{\Delta} \beta_{\mathbf{T}}+\Delta \mathbf{G}_{\mathbf{s}} \underline{\underline{\mathbf{M}}}_{s a}+\boldsymbol{\Delta} \mathbf{R}_{\mathbf{s}} \frac{\partial \underline{\underline{\mathbf{M}}}_{s a}}{\partial R_{s}} \\
& \left.+\Delta \mathbf{Z}_{\mathbf{s}} \frac{\partial \underline{\underline{\underline{M}}}_{s a}}{\partial Z_{f}}\right) \mathbf{I}_{a}
\end{aligned}
$$

In the Eqs. 5-7, $\boldsymbol{\Delta} \mathbf{G}_{\mathbf{a}}, \boldsymbol{\Delta} \mathbf{G}_{\mathbf{f}}, \boldsymbol{\Delta} \mathbf{G}_{\mathbf{m}}$ and $\boldsymbol{\Delta} \mathbf{G}_{\mathbf{s}}$ are calibration errors in the PF circuit currents, the flux loops, the magnetic field probes and the SLs; $\boldsymbol{\Delta} \beta_{\mathbf{T}}$ is the variation of the current distribution in the T coils; $\boldsymbol{\Delta} \mathbf{R}_{\mathbf{c}}$ and $\boldsymbol{\Delta} \mathbf{Z}_{\mathbf{c}}$ are the errors in the radius and in the vertical position of the PF coils; $\Delta \mathbf{R}_{\mathbf{f}}, \boldsymbol{\Delta} \mathbf{Z}_{\mathbf{f}}, \boldsymbol{\Delta} \mathbf{R}_{\mathbf{m}}, \boldsymbol{\Delta} \mathbf{Z}_{\mathbf{m}}, \boldsymbol{\Delta} \mathbf{R}_{\mathbf{s}}$ and $\boldsymbol{\Delta} \mathbf{Z}_{\mathbf{s}}$ are the errors in the position of the flux loops, magnetic probes and the virtual flux loops used to characterize the SLs (see Fig. $1-2$ ); $\Delta \alpha_{\mathbf{m}}$ is the error in the orientation of the magnetic probes; $\boldsymbol{\Delta} \mathbf{X}_{\mathbf{c}}$ and $\boldsymbol{\Delta} \mathbf{Y}_{\mathbf{c}}$ are the shifts of the PF coils along the $\mathrm{X}$ axis and the $\mathrm{Y}$ axis and $\boldsymbol{\Delta} \alpha_{\mathbf{c x}}$ and $\boldsymbol{\Delta} \alpha_{\mathbf{c y}}$ the tilts around the same axis (see APPENDIX A).

Each of the error parameters produce a difference between the measured quantities and the expected values that is estimated as a first order expansion of the relative Green's function with respect to the considered parameter.

The discrepancy found in Eqs. 2-4 are used as an input for the system of Eqs. 5-7. The error parameters are then estimated by inverting the system. This estimation can then be used as a set of correction factors for the geometrical and calibration parameters. Even if they represents a large number of parameters, $\left(\boldsymbol{\Delta} \mathbf{G}_{\mathbf{a}}{ }^{1 \times 19}, \boldsymbol{\Delta} \mathbf{R}_{\mathbf{c}}{ }^{1 \times 26}\right.$, $\Delta \mathbf{Z}_{\mathbf{c}}{ }^{1 \times 26}, \boldsymbol{\Delta} \mathbf{X}_{\mathbf{c}}{ }^{1 \times 26}, \boldsymbol{\Delta} \mathbf{Y}_{\mathbf{c}}{ }^{1 \times 26}, \boldsymbol{\Delta} \alpha_{\mathbf{c x}}{ }^{1 \times 26}, \boldsymbol{\Delta} \alpha_{\mathbf{c y}}{ }^{1 \times 26}, \boldsymbol{\Delta} \mathbf{G}_{\mathbf{m}}{ }^{1 \times 76}, \boldsymbol{\Delta} \mathbf{R}_{\mathbf{m}}{ }^{1 \times 76}, \boldsymbol{\Delta} \mathbf{Z}_{\mathbf{m}}{ }^{1 \times 76}$, $\Delta \alpha_{\mathbf{m}}{ }^{1 \times 76}, \boldsymbol{\Delta} \mathbf{G}_{\mathbf{f}}{ }^{1 \times 48}, \Delta \mathbf{R}_{\mathbf{f}}{ }^{1 \times 48}, \Delta \mathbf{Z}_{\mathbf{f}}{ }^{1 \times 48}, \Delta \mathbf{G}_{\mathbf{s}}{ }^{1 \times 24}, \Delta \mathbf{R}_{\mathbf{s}}{ }^{1 \times 6}, \Delta \mathbf{Z}_{\mathbf{s}}{ }^{1 \times 6}, \Delta \beta_{\mathbf{T}}{ }^{1 \times 1}: 660$ parameters in total), the inversion of the system is feasible since typically each signal must be corrected for 3 or 4 parameters but provides 19 independent measurements, one from each powered PF circuit, giving a total number of 3173 measurements $\left(\Psi_{f}^{48 \times 19}\right.$, $\mathbf{b}_{m}^{76 \times 19}, \boldsymbol{\Psi}_{s}^{24 \times 19}$ and $\left.\mathbf{I}_{c}^{19 \times 19}\right)$.

The correction parameters are determined in the least square sense by minimizing a cost function combining the residual errors:

$$
\begin{aligned}
\chi^{2} & =\sum_{f, k} w_{\Psi_{f}}^{2} \Delta \Psi_{f k}^{2}+\sum_{m, k} w_{b_{m}}^{2} \Delta b_{m k}^{2} \\
& +\sum_{s, k} w_{\Psi_{s}}^{2} \Delta \Psi_{m k}^{2}+\sum_{p} w_{p}^{2} \Delta P_{p}^{2}
\end{aligned}
$$


where $k$ is the data point index and $w_{\Psi_{f}}^{2}, w_{b_{m}}^{2}$ and $w_{\Psi_{s}}^{2}$ are weights, set to the inverse of the uncertainty of the considered measurement. To obtain a solution compatible with the expected values of the corrections, the results of the off-line calibration and the known position of the coils and sensors have to be added to the minimization process. This is done with the last term in Eq. (8), where $P_{p}$ is the considered parameter and $w_{p}^{2}$ is the corresponding weight, chosen to be the inverse of the expected error.

The results of this minimization process are shown in Fig. 4, where the differences between the measured quantities and the expected values computed from Eqs. 2-4 (blue dots) are compared to the differences computed from the Eqs. 5-7, using the derived corrections in the calibration and the location of the magnetic signals (black line). The good agreement between the two traces verifies the corrections of the parameters obtained from the minimization process.

The only source of non-axisymmetric errors in Eq. 7 is the wrong alignment of the PF coils. Therefore, the computed asymmetric error is only due to the misalignment between the PF coils and the vacuum vessel where the SLs are mounted. This corresponds to the real non-axisymmetric error only if the vacuum vessel is aligned with the toroidal field produced by the toroidal field coils. Unfortunately, since the toroidal field around the vacuum vessel is not measured, it is not possible to verify this assumption. Nevertheless, the mechanical uncertainty on the position of the toroidal field coils with respect to the vacuum vessel is of the order of a millimeter. Moreover, the absence of asymmetries on the TCV carbon tiles where the divertor strike points are usually located suggests a good alignment between the vacuum vessel and the toroidal magnetic field.

\section{Poloidal field coil irregularities}

The solution of Eq. 8 described above and its consequences are detailed in this section.

In Fig. 5, the axisymmetric $n=0$ errors in the PF coils $\left(\Delta G_{a}, \Delta R_{c}\right.$ and $\left.\Delta Z_{c}\right)$ are plotted together with the $n=1$ position errors $\left(\Delta X_{c}, \Delta Y_{c}, \Delta E_{c}=\sqrt{\Delta X_{c}^{2}+\Delta Y_{c}^{2}}, \Delta \alpha_{c x}\right.$ and $\left.\Delta \alpha_{c y}\right)$. The error on the position of the PF coils is of few millimeters. Only coils $\mathrm{C}$ and $\mathrm{D}$ have a larger error $(\sim 2 \mathrm{~cm})$. The position of the central column, where the $\mathrm{A}$ and $\mathrm{E}$ coils are located, seems to be shifted by $5 \mathrm{~mm}$ with respect to the vacuum vessel. The $E$ coils are also all shifted relatively to the vacuum vessel, suggesting the possibility of a tilt between the central column and the vacuum vessel of $0.25^{\circ}$.

The error bars of the estimated errors are different for each parameter and each PF coil. This is due to the non-uniform coupling between the PF coils and the magnetic sensors and the different sensitivity of the considered parameter.

The variation of flux on the SLs due to a shift of a PF coil in the $\mathrm{X}$ direction or a tilt around the $\mathrm{X}$ axis is plotted for each coil in Fig. 6 . The flux variation due to a tilt of a coil is smaller than the one created by a rigid movement in one direction, consequently the error bars for the parameters $\Delta \alpha_{c x}$ and $\Delta \alpha_{c y}$ in Fig. 5 are larger. The different order of magnitude between the flux induced by the $\mathrm{E}$ coils and the $\mathrm{F}$ coils (note that the data for the F-coils has been scaled by a factor 0.1 to fit on the plot) is justifying the larger error bars of the E coils. The F coils are closer to the SLs and therefore are better coupled with them. The error bars for the $\mathrm{O} 2$ and the $\mathrm{T}$ coil systems are even larger because besides the small coupling with the SLs, it is not possible to measure the effect of each single coil but only the magnetic field produced by the current distribution 
in the considered coil system. Notice that due to the large distance between the $\mathrm{O} 2$ and the $\mathrm{T}$ coils from the plasma region, the $n=1$ error field induced by their misalignment is relatively small (see Fig. 7).

On the right side of Fig. 5, the error on the SLs is plotted. The first plot is the error on the gain of each loop $\left(\Delta G_{s}\right)$. This is due to the calibration error of the amplifiers and to a wrong estimation of the toroidal extension of the SLs. The error of the radial and vertical position of the virtual poloidal flux loops $\left(\Delta R_{s}\right.$ and $\left.\Delta Z_{s}\right)$ is also shown in Fig. 5.

\section{Magnetic field error}

The error on the PF coils current calibration $\left(\Delta G_{a}\right.$ in Fig. 5) and on their radial and vertical position $\left(\Delta R_{c}\right.$ and $\Delta Z_{c}$ in Fig. 5) lead to an asymmetric $n=0$ error field inside the vacuum vessel. Its spacial distribution depends on the currents in the PF coils. In Fig. 8 (left), the radial and vertical $n=0$ error field is plotted for a typical TCV discharge. In Fig. 9 (left) the same error field distribution is shown at the breakdown time.

The non-asymmetric $n=1$ error field is computed form the shift and tilt irregularities of the PF coils $\left(\Delta X_{c}, \Delta Y_{c}, \Delta \alpha_{c x}\right.$ and $\Delta \alpha_{c y}$ in Fig. 5). In Fig. 8 (right) and in Fig. 9 (right), the distribution of the $n=1$ error field is represented for a TCV discharge and at the breakdown time.

Both the $n=0$ and the $n=1$ error fields are of the order of few $\mathrm{mT}$ and they are stronger in the proximity of the PF coils. At the breakdown time, the $n=0$ error field is responsible for a variation of the magnetic configuration and the $n=1$ error field may reduce the connection length in the null point region, where the magnetic field is smaller than $1 \mathrm{mT}$. However, at the breakdown time the magnetic field is strongly perturbed by the eddy currents induced in the vacuum vessel and in the magnetic structure $(\sim 20 \mathrm{mT})$. The distribution of the error field at the breakdown time in Fig. 9 is only representative of the contribution of the PF coils.

In order to estimate the topological effect of the error field on the magnetic equilibrium, the $n=1$ vacuum error field distribution inside the vessel is added to the equilibrium magnetic field. The resulting magnetic field configuration is illustrated by a Poincaré plot obtained by following a set of field lines and tracing their position after each toroidal turn (Fig. 10). An $n=1, m=1$ magnetic island is created at the normalized radius $\rho \sim 0.65$ ( $m$ being the poloidal mode number). The other $n=1, m>1$ modes are also present, but they have a smaller amplitude. Ergodization due to islands overlapping is present only at the very edge and it is therefore negligible.

The main components of the $n=1$ error magnetic field can be corrected by applying an asymmetric field of opposite sign. Based on the results of the presented magnetic calibration, a saddle coil system for the TCV tokamak has been proposed [15].

\section{Conclusion}

The magnetic system on TCV has been extended with a set of saddle loops used to measure the radial magnetic flux at different toroidal and vertical positions. From the calibration of the magnetic system, the errors on the position of the poloidal field coils 
and on the measured current have been computed and the associated error field inside the vacuum vessel for a typical TCV discharge has been estimated.

The sources of the $n=0$ error field have been identified and the geometrical and calibration parameters corrected to minimize it.

The $n=1$ perturbation introduced by the misalignment of the poloidal field coils as been added to the equilibrium field of a typical TCV discharge. The error field creates an $n=1, m=1$ magnetic island at $\rho \sim 0.65$. Islands with higher mode number have a smaller size and the consequent ergodization due to the island overlapping is negligible.

This work was supported in part by the Swiss National Science Foundation.

\section{Appendix A}

The following sign conventions are used in the article. The $Z$ axis points towards the top of the tokamak and the $Z=0$ plane coincides with the equatorial midplane; the $R$ axis points towards the outside; the toroidal angle $\varphi$ is positive in the counterclockwise direction, the $\varphi=0$ plane is chosen at the position of sector 16. The $X$ axis corresponds to the toroidal angle $\varphi=0$ and the $Y$ axis is located at sector $4\left(\varphi=90^{\circ}\right)$. The conventions are summarized in Fig. 11.

\section{References}

[1] F. Hofmann et al, Plasma Phys. Control. Fusion, 36 B277 (1994)

[2] D. D. Ryutov et al, Phys. Plasmas 14064502 (2007)

[3] F. Piras et al, Plasma Phys. Control. Fusion, 51055010 (2009)

[4] J-M. Moret et al, 23rd EPS Conf., Kiev (1996)

[5] R.J. Crossland et al, SOFT, London (1990)

[6] R.J. La Haye et al, Rev. Sci. Instrum., 62 (1991)

[7] J.L. Luxon et al, Fusion Engineering and Desing, 66-68 (2003)

[8] T.C. Hender et al, Nucl. Fusion 32 (1992)

[9] A.W. Morris et al, Phys. Fluids B 4 (1992)

[10] J.T. Scoville et al, Nucl. Fusion 31 (1991)

[11] R.J. La Haye et al, Phys. Fluids B 4 (1992)

[12] G.M. Fishpool et al, Nucl. Fusion 34 (1994)

[13] J.T. Scoville et al, Nucl. Fusion 43 (2003)

[14] J-M. Moret et al, Rev. Sci. Instrum., 66 (1998)

[15] J. Rossel et al, Plasma Phys. Control. Fusion (accepted) 


\section{C2}

D2

B2 flux loops

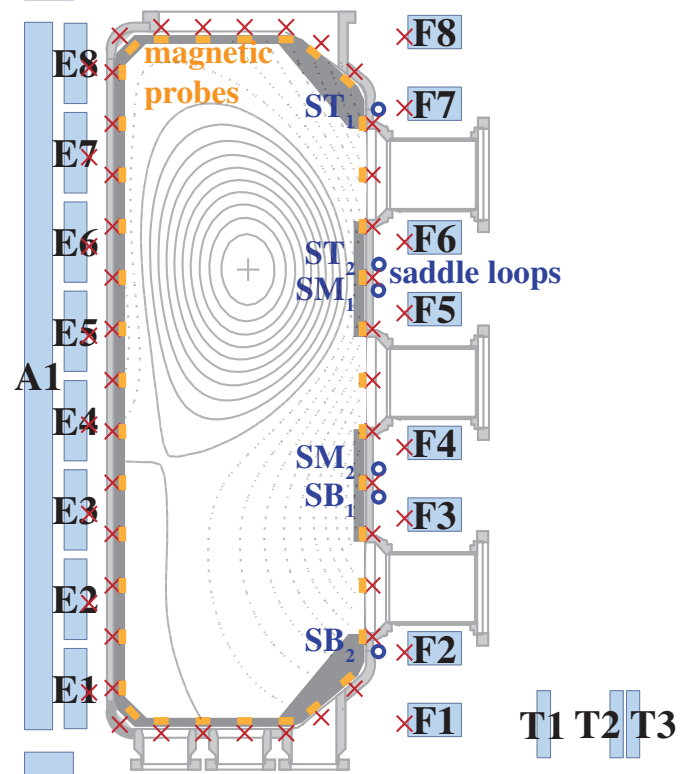

B1X

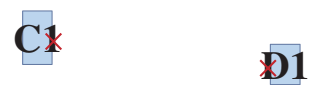

Figure 1: TCV poloidal cross section with a typical plasma equilibria showing the ohmic transformer coils A, B, C and D, the shaping coils E and F, the toroidal field coil connections $\mathrm{T}$, the poloidal flux loops (red crosses), the magnetic field probes (orange rectangles) and the saddle flux loops (blue circles). 


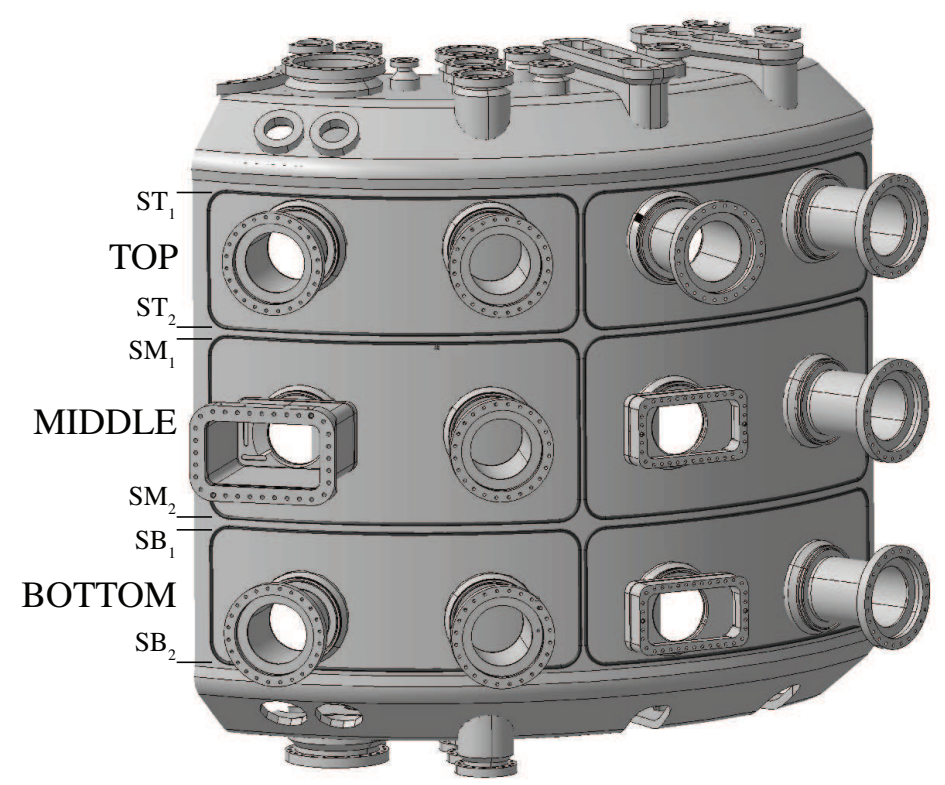

Figure 2: SLs location around the TCV vessel. Each SL covers two sectors toroidally and is vertically delimited by the virtual poloidal flux loops $\mathrm{ST}_{1,2}, \mathrm{SM}_{1,2}$ and $\mathrm{SB}_{1,2}$. They are located at three vertical positions (TOP, MIDDLE and BOTTOM).

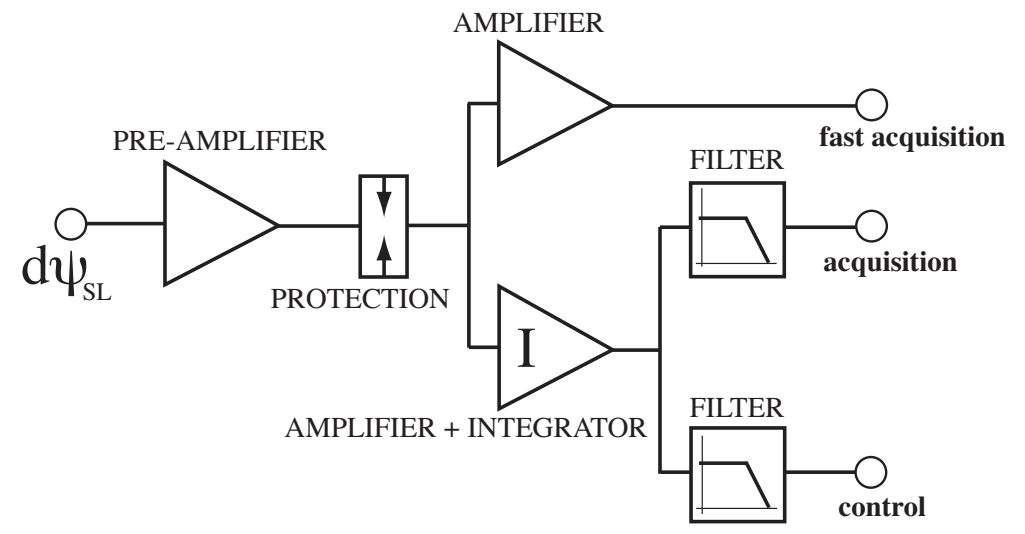

Figure 3: Block diagram of the electronic circuit used to acquire the SL signals. 


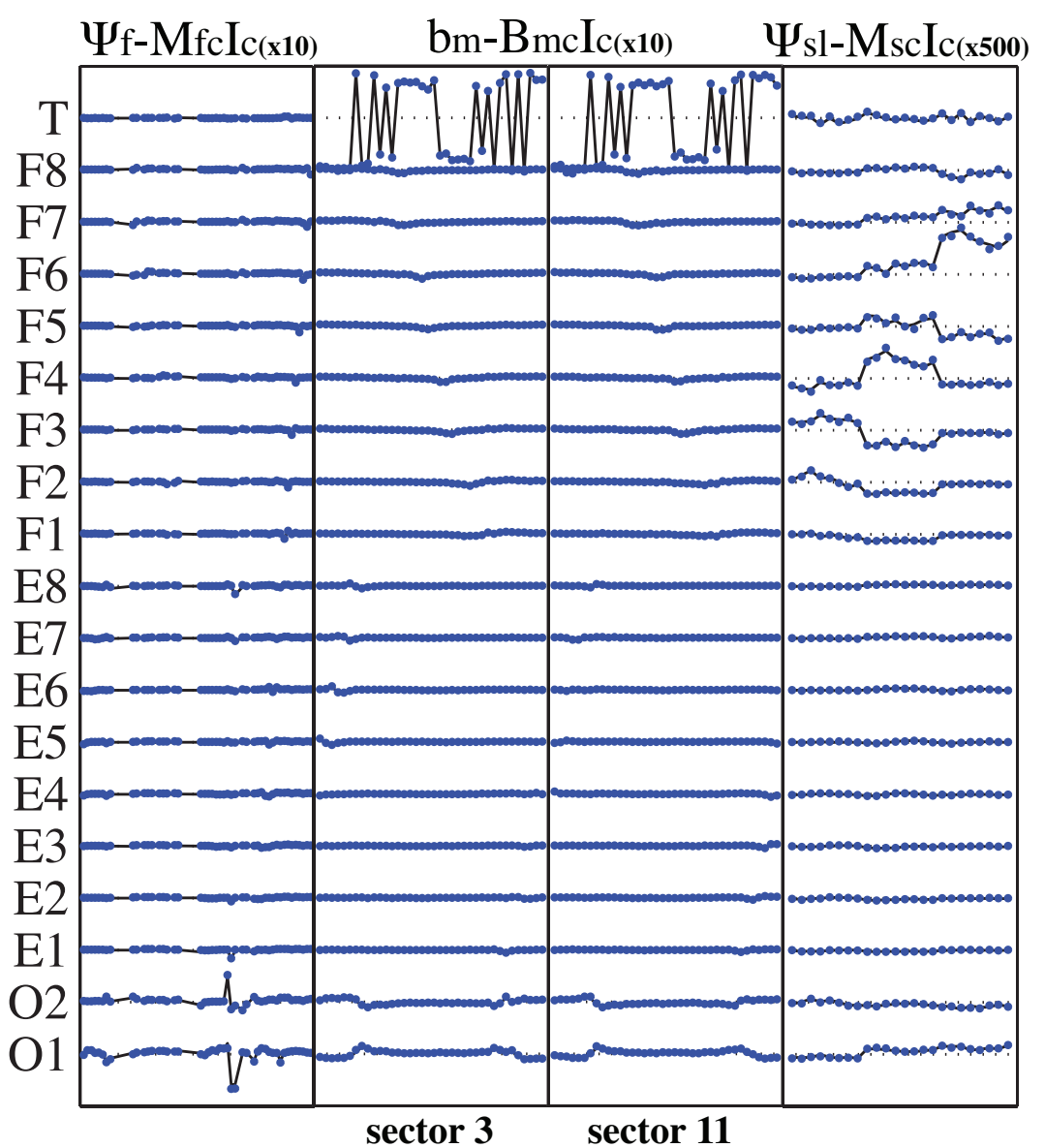

Figure 4: Differences between the measured magnetic quantities and the theoretical predictions for each coil as a function of the considered sensor. The blue dots show the differences with the measurements as defined in Eqs. 2-4, the solid lines are the differences computed from the derived errors on the calibration and the location of the magnetic signals, as in Eqs. 5-7 (black line). 

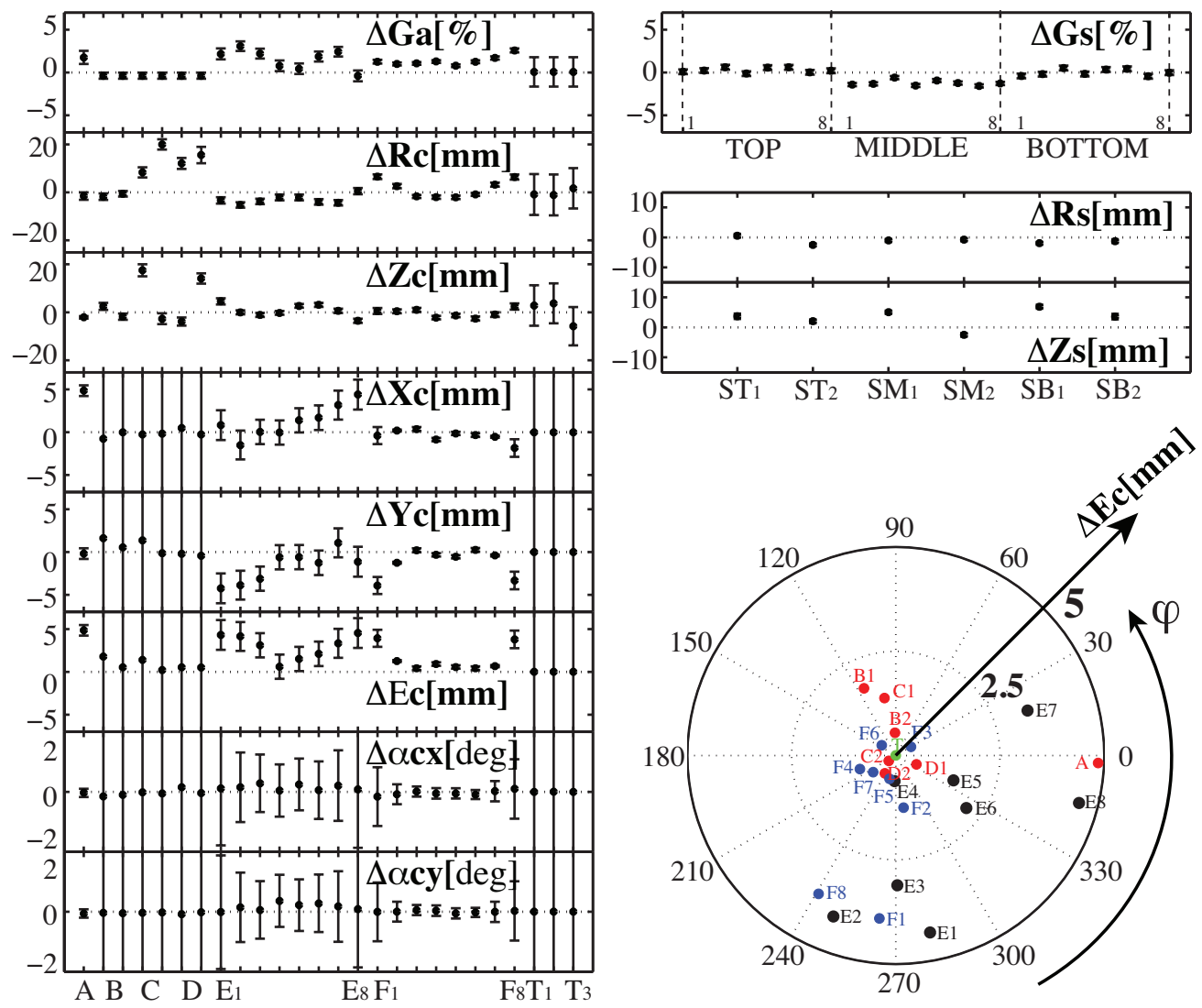

Figure 5: Corrections for the coil current calibration, the coil location, the SLs calibration and their location. 


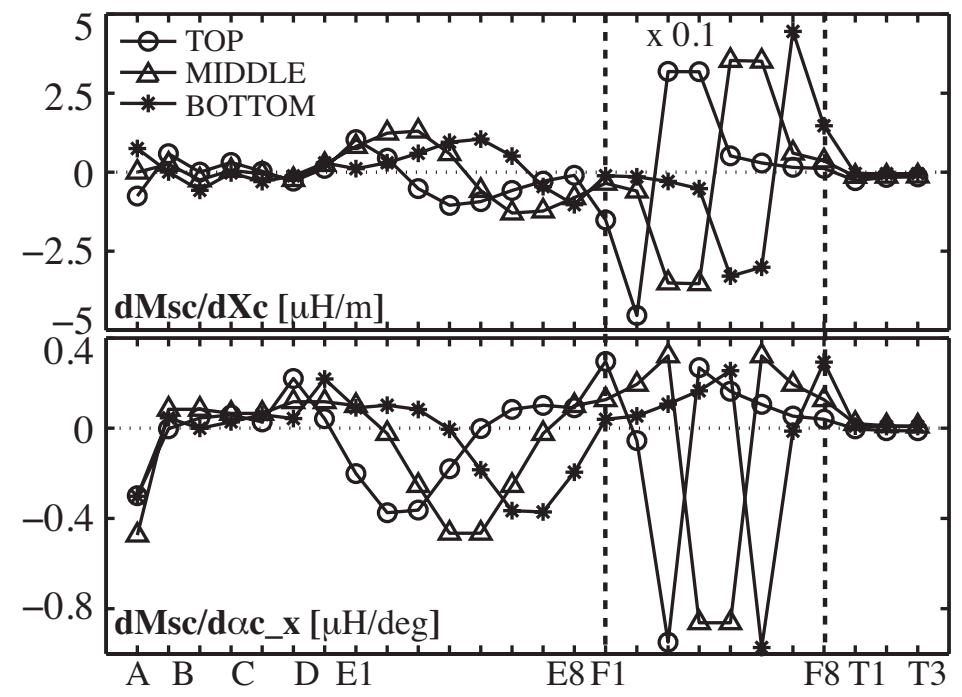

Figure 6: Variation of flux in the SLs due to a shift of a coil in the $\mathrm{X}$ direction or a tilt around the $\mathrm{X}$ axis (note that the data for the F-coils has been scaled by a factor 0.1 to fit on the plot).

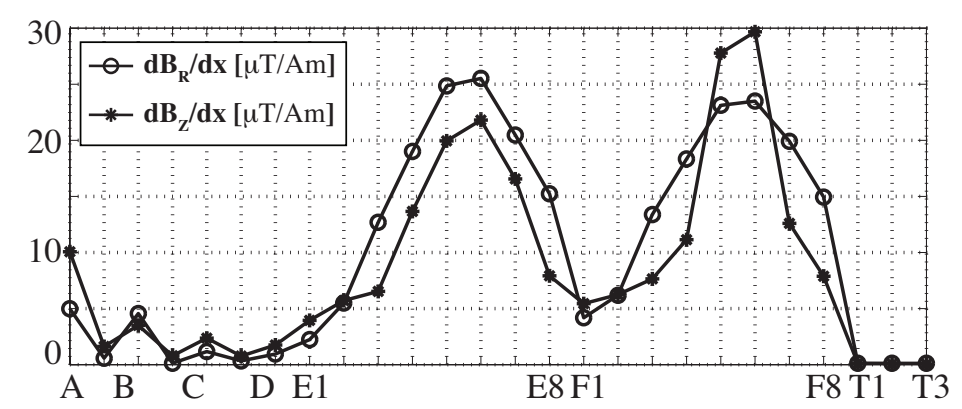

Figure 7: Absolute value of the radial and vertical $n=1$ error field per unit current and displacement of the PF coils averaged on the plasma region. 

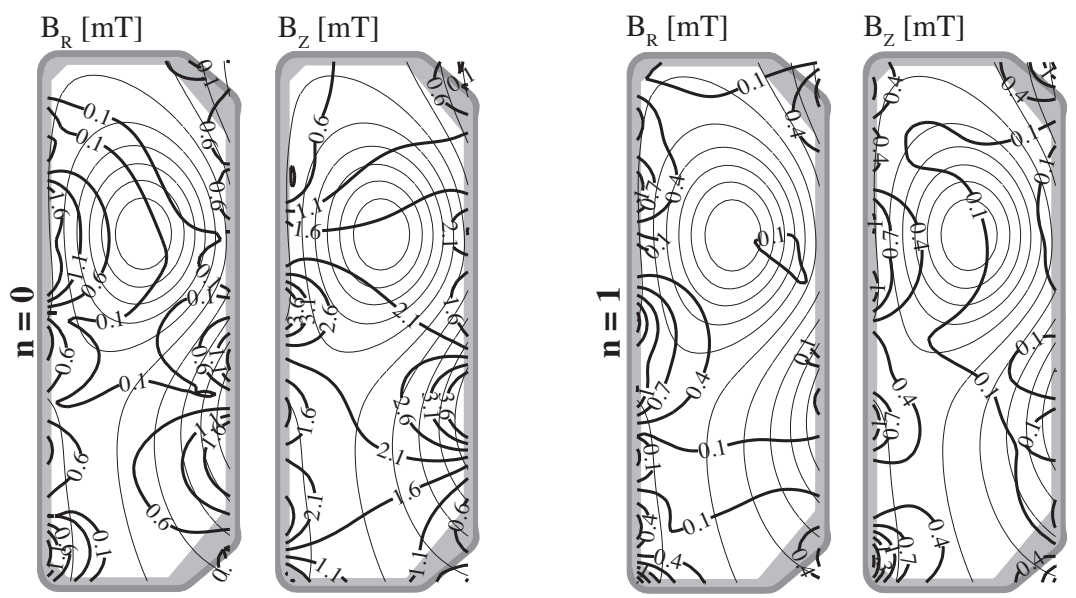

Figure 8: Radial and vertical $n=0$ (left) and $n=1$ (right) error field distribution inside the vacuum vessel for a typical TCV discharge (shot \#39109, $0.6 \mathrm{~s}$ ).
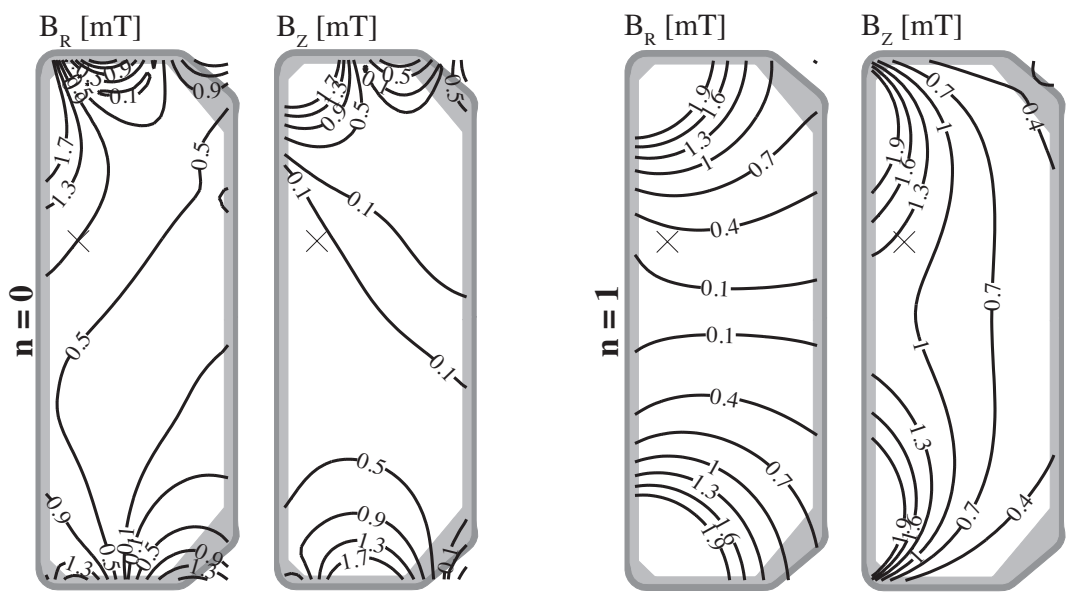

Figure 9: Radial and vertical $n=0$ (left) and $n=1$ (right) error field distribution inside the vacuum vessel for a typical TCV breakdown (shot \#39109, $0 \mathrm{~s}$ ). The cross point is the breakdown position. 


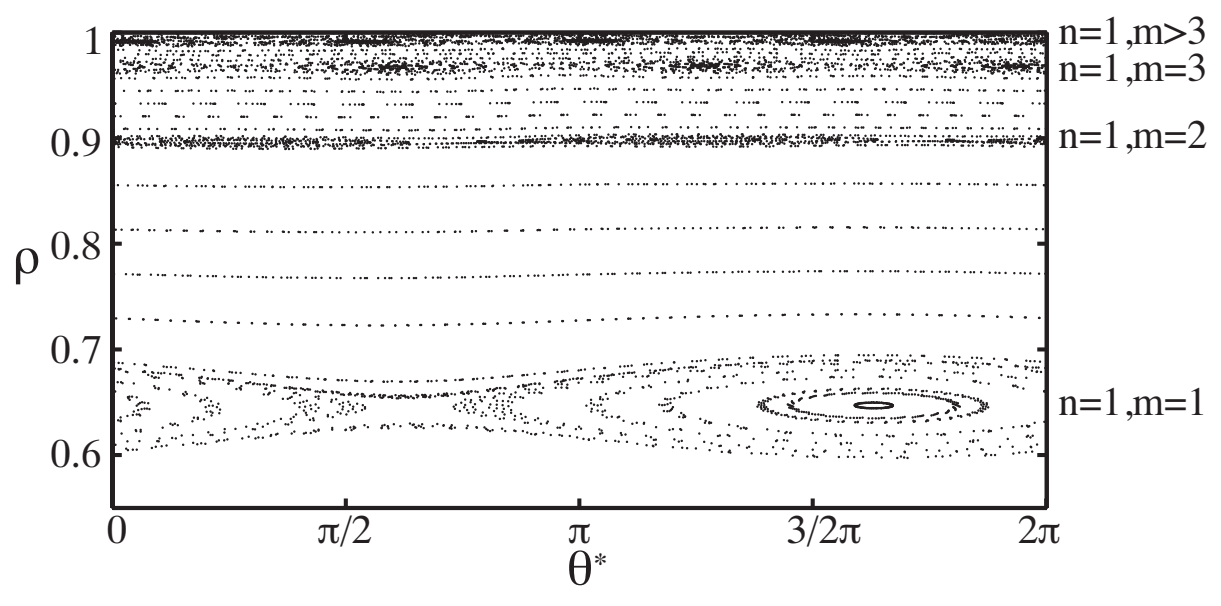

Figure 10: Poincaré plot of the vacuum error field added to the equilibrium magnetic field of a typical TCV discharge (shot \# 39109, 0.6s). $\rho=\sqrt{1-\psi / \psi_{0}}$ is the normalized plasma minor radius $(\psi$ is the poloidal flux) and $\theta^{\star}$ is the poloidal angle in straight field line coordinates.
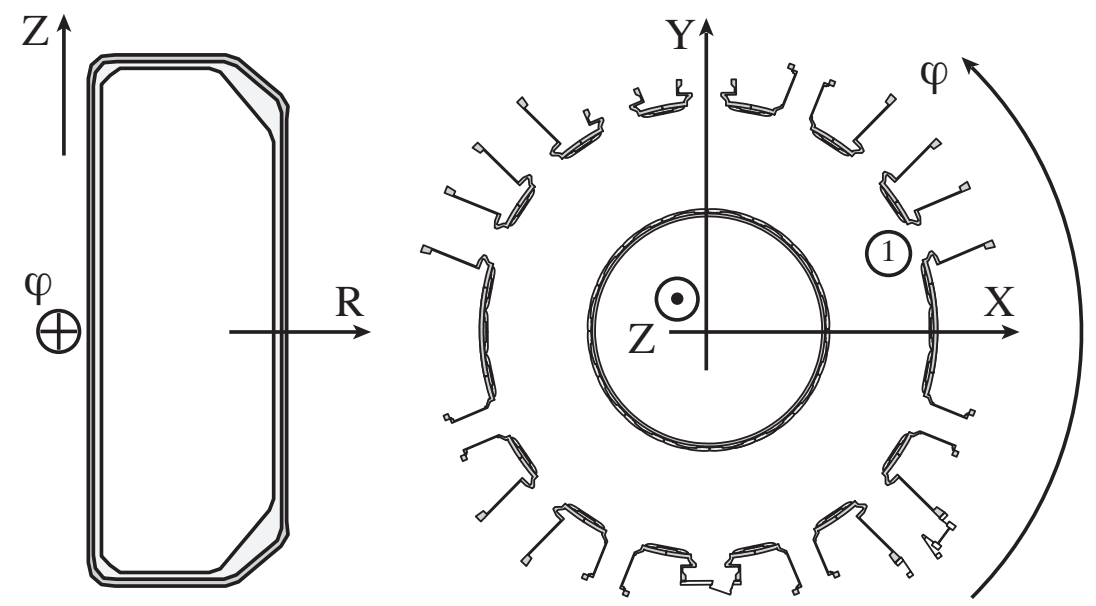

Figure 11: Sign convention. 